\title{
MOUSE: A MINI OBSERVATORY FOR UV SPACE EXPLORATION OF THE GALACTIC POLES AND EQUATOR
}

\author{
R. RAGAZZONI ${ }^{1}$, L. BUSON ${ }^{1}$, M. TURATTO ${ }^{1}$, A. BIANCHINI ${ }^{2}$, E. \\ CAPPELLARO $^{1,3}$, M. CECCONI ${ }^{4}$, F. LA FRANCA ${ }^{2}$, U. MUNARI ${ }^{1}$, S. \\ ORTOLANI $^{2}$, I. PORRO ${ }^{4}$, P. RAFANELLI ${ }^{2,4}$, R. STAGNI ${ }^{2,4}$, F. BERTOLA ${ }^{2,4}$, P.L. \\ BERNACCA $^{2,4}$ \\ ${ }^{1}$ Astronomical Observatory of Padova, Italy \\ ${ }^{2}$ Department of Astronomy, University of Padova, Italy \\ ${ }^{3}$ European Southern Observatory, La Silla, Chile \\ ${ }^{4}$ Center of Studies and Activities for Space 'G. Colombo', University of Padova, \\ Italy
}

\begin{abstract}
The small orbiting observatory MOUSE is described in its essential features. Insights in the optical solution (a 2 mirrors 3 reflections telescope using pure hyperbolical surfaces, no aspherics) are given which fit such type of optical layout to scientific requirements. An in-flight recentering of the photons collected by a solar-blind photon counter device will allow for an equivalent angular resolution of 2-3 arcsec over a field-of-view exceeding one square degree. The capabilities of the proposed satellite (imaging, low resolution grisms, polarizers) are described. The choice of the selected sky areas (the two Galactic Poles, the galactic equator and some Bulge Windows) is justified in terms of forefront astrophysical scientific objectives requiring imaging in UV over large fields. The science comprises UV analysis of a complete sample of QSOs, stellar population in galaxies, White Dwarfs, Globular and Open Clusters and OB Associations. In addition a plan for the early UV detection of Supernova events is described.
\end{abstract}

It has been proposed to ESA to fly a Mini Observatory for Ultraviolet Space Exploration (MOUSE) in order to carry on a Core Scientific Programme (CSP) that can solely be achieved from space in the satellite ultraviolet over wide fields.

The CSP shall be eventually finalized by screening of forefront scientific astrophysical problems which require imaging in selected spectral bands through the range $1250-3000 \AA$. A number of Core Scientific Objectives (CSO) have already been presented to ESA: a) stellar populations in galaxies; b) survey of QSOs; c) supernovae; d) white dwarfs in galactic fields; e) globular clusters; $f$ ) open clusters and OB associations; $g$ ) exotica in galactic fields; $h$ ) cataclysmic variables; i) looking through galactic windows; $j$ ) the magellanic clouds.

The CSP will be of PI-type and shall be accomplished in less than 2.5 years. There will be one PI for each of the CSOs which will be entitled to be part of the CSP. It is believed that each of the CSOs requires a large aggregation of scientists in order to effectively be dealt with and therefore this project may encourage international cooperation among interested astronomers.

The images obtained for the CSP can also be exploited by scientists in a Guest Investigator Programme (GIP) on the basis of an announcement of opportunity for scientific purposes different from those of the CSO. 
The sample CSP itemized above can be accomplished (see Fig. 1) by surveying the equatorial belt of the galaxy, some bulge strips, and probing the extragalactic environment by looking through the galactic poles.

After the CSP has been performed by ESA as part of the mandatory programme, a Guest Observatory Programme (GOP) can be carried on an optional basis following solicited research proposals from the interested astronomical communities which shall pay for satellite and scientific operations. To the purpose of GOP the satellite has been designed for a minimum of 5 years lifetime.

The MOUSE payload consists of a 2 mirror 3 reflections $F / 2$ telescope having a primary mirror of about $60 \mathrm{~cm}$ (see Fig. 2 and Table 1). On the focal plane, behind the secondary mirror, two filter wheels are implemented in front of a photon counting detector. The wheels will allow imaging in three wide bands (centered around $1500 \AA, 2200 \AA$ and $2800 \AA$ ), polarimetry and low resolution grism spectroscopy.

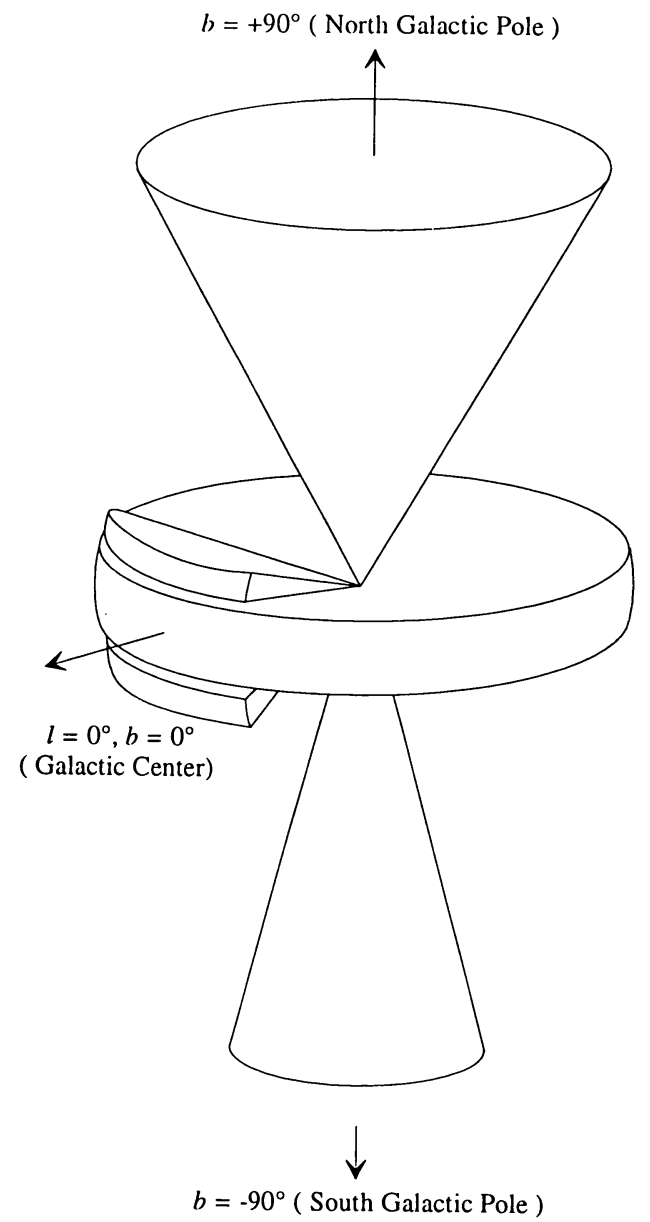

Figure 1. The sky coverage for MOUSE. 


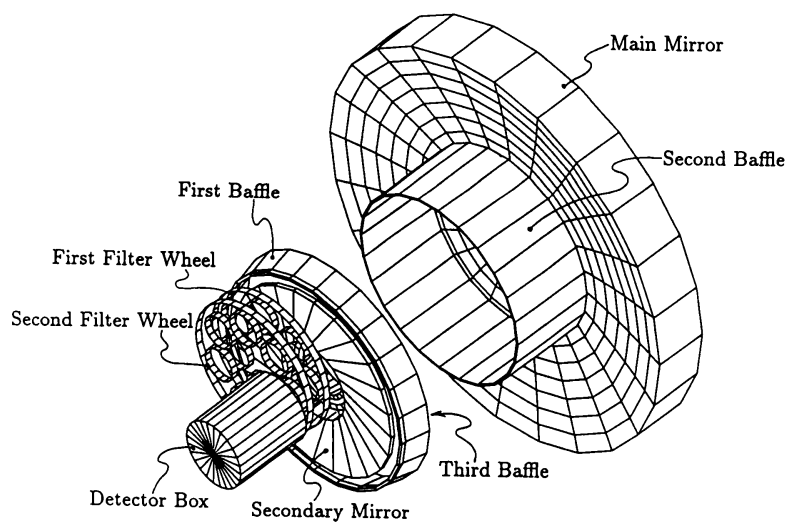

Figure 2. The overall optical layout of MOUSE.

Table 1. MOUSE telescope main characteristics

\begin{tabular}{lc}
\hline \multicolumn{1}{c}{ Configuration } & 2 mirrors 3 reflections \\
\hline \hline Aperture & $600 \mathrm{~mm}, \varepsilon \approx 0.5$ \\
Focal Length & $f=1200 \mathrm{~mm}$ \\
Focal Ratio & $F /=2$ \\
Field Size & $\approx 2^{\circ}$ in diameter \\
Image Quality on Focal Plane & better than $\approx 2^{\prime \prime}(*)$ \\
Mirror Coating & ${\mathrm{Al}-\mathrm{MgF}_{2}}^{*}$ \\
\hline (*) At 80\% GEE &
\end{tabular}

Since the expected jitter of the spacecraft $(\approx 30 \mathrm{arcsec})$ would spoil the optical resolution of the telescope, a special CCD tracker, working in the visible bandwidth, collects the light fed by a small mirror close to the focal plane in order to allow an accurate attitude knowledge for onboard image recentering. The image quality, obtained via in-flight recentering, should be of the order of 1 to 3 arcsec. Wide band (600A்), limiting magnitudes for detection of hot sources $\left(T=3.5\right.$...5.0 $\left.\times 10^{\AA} \mathrm{K}\right)$ at $1500 \AA$ are close to $V=24$...25 (30 m exposure time) for the case of no reddening and general UV background. They reduce to $V \approx 21$ for $E(B-V)=1$.

The proposed orbit (allowing a $>5$ years lifetime) is elliptical with $\approx 550 \mathrm{Km}$ perigee altitude and $\approx 800 \mathrm{Km}$ apogee altitude, inclined on the equator of $41^{\circ}$. Villafranca (Spain) is proposed as ground station.

The satellite mass is expected to be less than $320 \mathrm{Kg}$ (including the $\mathrm{P} / \mathrm{L}$ ) and may be launched into the selected orbit by a Pegasus class launcher. The launch date is foreseen in 1999. 